\title{
CICATRIZAÇÃO DE FERIDAS
}

\author{
Wound healing
}

Antonio Carlos Ligocki CAMPOS, Alessandra BORGES-BRANCO, Anne Karoline GROTH

ABCDDV/525

Campos ACL, Borges-Branco A, Groth AK. Cicatrização de feridas. ABCD Arq Bras Cir Dig 2007;20(1):51-8.

RESUMO - Introdução - A cicatrização de feridas consiste em perfeita e coordenada cascata de eventos que culminam com a reconstituição tecidual. O processo cicatricial é comum a todas as feridas, independe do agente que a causou. O processo de cicatrização é dividido didaticamente em três fases: inflamatória, proliferação ou granulação e remodelamento ou maturação. O colágeno é a proteína mais abundante no corpo humano e também é o principal componente da matriz extracelular dos tecidos. Estrutura-se numa rede densa e dinâmica resultante da sua constante deposição e reabsorção. O tecido cicatricial é resultado da interação entre sua síntese, fixação e degradação. Existem várias maneiras de avaliar a cicatrização das feridas. Os métodos mais utilizados atualmente são a tensiometria, a densitometria e morfometria do colágeno, a imunoistoquímica e, mais recentemente, a dosagem de fatores de crescimento. Métodos - Foram revisadas todas as dissertações de mestrado e teses de doutorado realizadas na linha de pesquisa "Cicatrização de órgãos e tecidos" do programa de Pós-Graduação em Clínica Cirúrgica da Universidade Federal do Paraná. Foi realizada, também, revisão atualizada da literatura internacional no Pubmed (www.pubmed.com) e nacional (www.bases.bireme.br). Conclusão - A cicatrização é um processo complexo, que começou a ser entendido em maior amplitude nos últimos anos. Recentes pesquisas têm sido direcionadas para atuar na modulação da cicatrização a nível molecular, na tentativa de evitar completamente cicatrizes patológicas.

DESCRITORES - Cicatrização de feridas.

\section{INTRODUÇÃO}

O processo cicatricial é comum a todas as feridas, independentemente do agente que a causou, é sistêmico e dinâmico e está diretamente relacionado às condições gerais do organismo ${ }^{12}$. A cicatrização de feridas consiste em perfeita e coordenada cascata de eventos celulares, moleculares e bioquímicos que interagem para que ocorra a reconstituição tecidual. Os mecanismos da cicatrização em seqüência ordenada de eventos foram descritos por Carrel em $1910^{13}$, e divididos posteriormente em cinco elementos principais: inflamação, proliferação celular, formação do tecido de granulação, contração e remodelação da ferida ${ }^{39}$. Recentemente, Clark reclassificou esse processo em três fases divididas, didaticamente, em: fase inflamatória, fase de proliferação ou de granulação e fase de remodelação ou de maturação $0^{15}$.

A lesão tecidual - estímulo inicial para o processo de cicatrização - coloca elementos sangüíneos em contato com o colágeno e outras substâncias da matriz extracelular, provocando degranulação de plaquetas e ativação das cascatas de coagulação e do complemento. Com isso, há liberação de vários mediadores vasoativos e quimiotáticos que conduzem o processo cicatricial mediante atração de células inflamatórias para a região da ferida ${ }^{61}$.

Trabalho realizado no Departamento de Cirurgia do Setor de Ciências da Saúde da Universidade Federal do Paraná e Pós- Graduação em Clínica Cirúrgica da Universidade Federal do Paraná, Curitiba, PR, Brasil.

Endereço para correspondência: Antonio Carlos L. Campos. e-mail: accampos@hc.ufpr.br

\section{MÉTODOS}

Foram revisadas as dissertações de mestrado e teses de doutorado realizadas na linha de pesquisa "Cicatrização de órgãos e tecidos" do programa de Pós-Graduação em Clínica Cirúrgica da Universidade Federal do Paraná. Foi realizada, também, revisão atualizada da literatura internacional no PubMed (www.pubmed.com) e nacional (www.bireme.br), utilizando os descritores: cicatrização de feridas; colágeno; tensiometria. Os artigos considerados pertinentes foram utilizados e constam nas referências deste trabalho.

\section{REVISÃO DA LITERATURA}

\section{Fases da cicatrização}

\section{Fase inflamatória}

Esta fase se inicia imediatamente após a lesão, com a liberação de substâncias vasoconstritoras, principalmente tromboxana A2 e prostaglandinas, pelas membranas celulares. O endotélio lesado e as plaquetas estimulam a cascata da coagulação. As plaquetas têm papel fundamental na cicatrização. Visando a hemostasia, essa cascata é iniciada e grânulos são liberados das plaquetas, as quais contêm fator de crescimento de transformação beta - TGF- $\beta$ (e também fator de crescimento derivado das plaquetas [PDGF], fator de crescimento derivado dos fibroblastos [FGF], fator de crescimento epidérmico [EGF], prostaglandinas e trom- 
boxanas), que atraem neutrófilos à ferida ${ }^{11}$. O coágulo é formado por colágeno, plaquetas e trombina, que servem de reservatório protéico para síntese de citocinas e fatores de crescimento, aumentando seus efeitos. Desta forma, a resposta inflamatória se inicia com vasodilatação e aumento da permeabilidade vascular, promovendo a quimiotaxia (migração de neutrófilos para a ferida).

Neutrófilos são as primeiras células a chegar à ferida, com maior concentração 24 horas após a lesão. São atraídos por substâncias quimiotáticas liberadas por plaquetas. Os neutrófilos aderem à parede do endotélio mediante ligação com as selectinas (receptores de membrana). Neutrófilos produzem radicais livres que auxiliam na destruição bacteriana e são gradativamente substituídos por macrófagos ${ }^{11}$.

Os macrófagos migram para a ferida após 48 - 96 horas da lesão, e são as principais células antes dos fibroblastos migrarem e iniciarem a replicação. Têm papel fundamental no término do desbridamento iniciado pelos neutrófilos e sua maior contribuição é a secreção de citocinas e fatores de crescimento, além de contribuírem na angiogênese, fibroplasia e síntese de matriz extracelular ${ }^{11}$, fundamentais para a transição para a fase proliferativa.

\section{Fase proliferativa}

A fase proliferativa é constituída por quatro etapas fundamentais: epitelização, angiogênese, formação de tecido de granulação e deposição de colágeno. Esta fase tem início ao redor do $4^{\circ}$ dia após a lesão e se estende aproximadamente até o término da segunda semana. A epitelização ocorre precocemente. Se a membrana basal estiver intacta, as células epiteliais migram em direção superior, e as camadas normais da epiderme são restauradas em três dias. Se a membrana basal for lesada, as células epiteliais das bordas da ferida começam a proliferar na tentativa de restabelecer a barreira protetora ${ }^{32}$.

A angiogênese é estimulada pelo fator de necrose tumoral alfa (TNF- $\alpha$ ), e é caracterizada pela migração de células endoteliais e formação de capilares, essencial para a cicatrização adequada.

A parte final da fase proliferativa é a formação de tecido de granulação. Os fibroblastos e as células endoteliais são as principais células da fase proliferativa. Os fibroblastos dos tecidos vizinhos migram para a ferida, porém precisam ser ativados para sair de seu estado de quiescência. $O$ fator de crescimento mais importante na proliferação e ativação dos fibroblastos é o PDGF ${ }^{12}$. Em seguida é liberado o TGF- $\beta$, que estimula os fibroblastos a produzirem colágeno tipo I e a transformarem-se em miofibroblastos, que promovem a contração da ferida.

Entre os fatores de crescimento envolvidos no processo cicatricial podem ser citados o PDGF, que induz a proliferação celular, a quimiotaxia e a síntese matricial; o fator epidérmico, que estimula a epitelização; o fator transformador alfa, responsável pela angiogênese e pela epitelização; o fator fibroblástico, que estimula a proliferação celular e angiogênese e o fator transformador beta, responsável pelo aumento da síntese matricial ${ }^{61}$ (Tabela 1).
TABELA 1 - Principais fatores de crescimento envolvidos no processo cicatricial

\begin{tabular}{|c|c|c|}
\hline $\begin{array}{l}\text { Fator de } \\
\text { Crescimento }\end{array}$ & Origem & Função \\
\hline TNF- $\alpha$ & Monócitos e linfócitos & $\begin{array}{l}\text { Proliferação de fibroblastos. } \\
\text { Quimiotaxia para neutrófilos e } \\
\text { macrófagos }\end{array}$ \\
\hline TGF- $\alpha$ & $\begin{array}{l}\text { Matriz extracelular da } \\
\text { ferida cirúrgica }\end{array}$ & $\begin{array}{l}\text { Proliferação celular } \\
\text { Estimula a epitelização }\end{array}$ \\
\hline TGF- $\beta$ & $\begin{array}{l}\text { Plaquetas, matriz } \\
\text { extracelular da ferida } \\
\text { cirúrgica }\end{array}$ & $\begin{array}{l}\text { Mitogênicos para fibroblastos } \\
\text { Formação de tecido de granulação }\end{array}$ \\
\hline PDGF & Plaquetas & $\begin{array}{l}\text { Quimiotaxia para neutrófilos, } \\
\text { monócitos e fibroblastos. } \\
\text { Proliferação de fibroblastos e a } \\
\text { produção de matriz extracelular }\end{array}$ \\
\hline VEGF & $\begin{array}{l}\text { Queratinócitos e } \\
\text { macrófagos }\end{array}$ & $\begin{array}{l}\text { Angiogênese e a proliferação de } \\
\text { células endoteliais }\end{array}$ \\
\hline IL-1 & Mononucleares & Proliferação de fibroblastos \\
\hline
\end{tabular}

Legenda: TNF- $\alpha$, fator de necrose tumoral alfa; TGF- $\alpha$, fator de crescimento de transformação alfa; TGF- $\beta$, fator de crescimento de transformação beta; PDGF, fator de crescimento derivado das plaquetas; VEGF, fator de crescimento derivado do endotélio vascular; IL-1, interleucina 1

Adaptado de Broughton and Attinger, 2006 ${ }^{12}$.

\section{Fase de maturação ou remodelamento}

A característica mais importante desta fase é a deposição de colágeno de maneira organizada, por isso é a mais importante clinicamente. O colágeno produzido inicialmente é mais fino do que o colágeno presente na pele normal, e tem orientação paralela à pele. Com o tempo, o colágeno inicial (colágeno tipo III) é reabsorvido e um colágeno mais espesso é produzido e organizado ao longo das linhas de tensão. Estas mudanças se refletem em aumento da força tênsil da ferida. A reorganização da nova matriz é um processo importante da cicatrização. Fibroblastos e leucócitos secretam colagenases que promovem a lise da matriz antiga. A cicatrização tem sucesso quando há equilíbrio entre a síntese da nova matriz e a lise da matriz antiga, havendo sucesso quando a deposição é maior. Mesmo após um ano a ferida apresentará um colágeno menos organizado do que o da pele sã, e a força tênsil jamais retornará a 100\%, atingindo em torno de $80 \%$ após três meses ${ }^{12}$.

\section{Importância do colágeno}

O colágeno é a proteína mais abundante do tecido conectivo em fase de cicatrização. As várias diferenças na sua composição química determinam as suas funções biológicas. Foram descritas até o presente 19 isoformas (tipos) de colágeno, codificadas por um único gene. Os tipos de fibras colágenas do tecido conjuntivo são helicoidais, com seqüência tripeptídica repetitiva composta de glicina-X-Y, sendo $X$ representada pela prolina e Y pela hidroxiprolina. A rica presença de glicina e prolina é necessária para que uma molécula madura de colágeno assuma a estrutura de tripla hélice ${ }^{44}$.

O colágeno tipo I é o mais freqüente, é sintetizado pelos fibroblastos, e é mais predominante em ossos e tendões. O tipo III é mais comumente encontrado em tecidos moles, como vasos sangüíneos, derme e fáscia. A derme sã contém aproximadamente $80 \%$ de colágeno tipo I e $20 \%$ de colágeno tipo 
III. Já o tecido de granulação expressa 30 a 40 \% de colágeno do tipo III, sendo considerado colágeno imaturo ${ }^{44}$.

A degradação do colágeno se inicia precocemente e é muito ativa durante o processo inflamatório. A sua digestão ocorre em ambiente extracelular e é mediada por colagenases específicas. São conhecidos quatro tipos de colagenases: as séricas (elastase, catepsina $\mathrm{C}$ e proteinase neutra) e as metaloproteinases. A atividade das colagenases é controlada por citocinas liberadas principalmente por células inflamatórias, endoteliais, fibroblastos e queratinócitos. A formação da matriz extracelular é, pois, resultante de um balanço entre a deposição (síntese) e degradação de colágeno.

O colágeno é o principal componente da matriz extracelular dos tecidos. Estrutura-se em rede densa e dinâmica resultante da sua constante deposição e reabsorção. A quantidade de colágeno modifica-se no cólon durante o pós-operatório de anastomoses colônicas, principalmente na região peri-anastomótica ${ }^{29}$. Essa variação é resultado da interação entre sua síntese, fixação e degradação no tecido cicatricial.

Na Figura 1, adaptada de Witte e Barbul ${ }^{61}$, pode-se observar a evolução da cicatrização das escaras, onde se observa a correlação entre o aparecimento do colágeno maduro e o aumento da resistência tênsil da ferida.
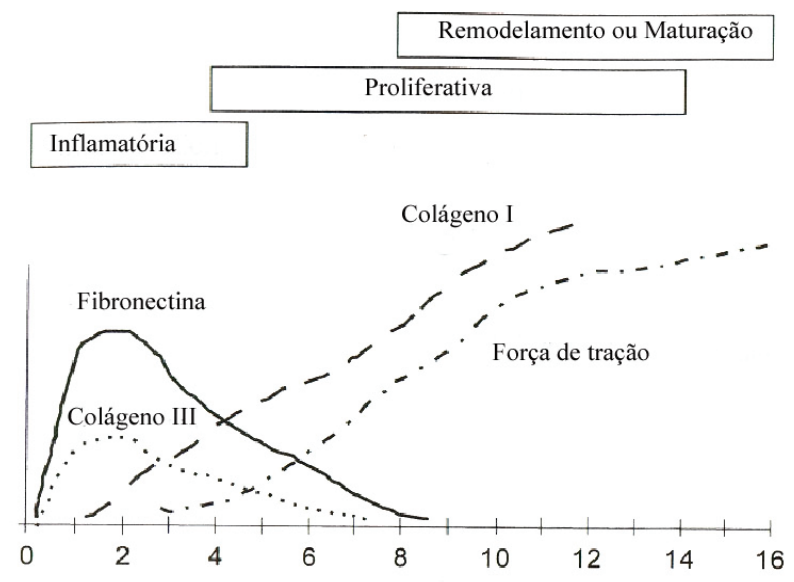

Adaptado de Witte \& Barbul, $1997^{61}$.

FIGURA 1 - Fases da cicatrização

\section{Fatores que influenciam a cicatrização}

\section{Fatores locais}

Vários fatores podem influenciar negativamente a cicatrização, dentre eles os principais são: isquemia, infecção, técnica cirúrgica ${ }^{19}$, corpo estranho e edema / pressão tecidual elevada ${ }^{2,3,34,52}$.

\section{Fatores sistêmicos}

Sistemicamente, diversos fatores podem afetar de alguma maneira a cicatrização, dentre eles pode-se citar: diabete melito, deficiências vitamínicas ${ }^{19}$, hipotiroidismo, doenças hereditárias (síndrome de Ehler-Danlos), alterações da coagulação, idade, trauma grave, queimaduras, sepse, insuficiência hepática e renal, insuficiência respiratória, tabagismo, radioterapia, desnutrição e o uso de corticosteróides, drogas antineoplásicas, ciclosporina A, colchicina e penicilamina ${ }^{11,12,19,32}$.

Estudos experimentais comprovaram que a nicotina compromete o processo cicatricial. Skinovsky estudou, em 2000 e 2005, a influência a injeção de nicotina subcutânea na cicatrização das anastomoses de intestino delgado em ratos, e concluiu que os animais que receberam nicotina apresentaram cicatrização deficiente quando comparados ao grupo controle ${ }^{47,48}$. Confirmando esses dados, Alves, em 2006, mostrou a cicatrização tecidual deficiente em ratos lactentes de mães que receberam nicotina subcutânea durante a gestação, representada pela redução da força de tração, da deposição de colágeno tipo III, da angiogênese e dos miofibroblastos ${ }^{4}$.

Diversos medicamentos também foram avaliados. Em 2003, Bonin avaliou o efeito dos inibidores de bomba de prótons na cicatrização das anastomoses gástricas e concluiu que, juntamente com o aumento do $\mathrm{pH}$ intragástrico, ocorre melhora da cicatrização, com aumento da força de ruptura e da deposição de colágeno tipo III $^{9,10}$. Ainda na avaliação da cicatrização das anastomoses gástricas, em 2004, Trubian estudou o efeito do octreotide subcutâneo e concluiu que ele não altera a cicatrização gástrica ${ }^{54}$. Entretando, Urdiales, em 2006, estudou o octreotide na cicatrização de anastomoses colônicas e concluiu que há melhora na deposição de colágeno tipo III, da força de ruptura e da força máxima de tração, melhorando a cicatrização ${ }^{56}$.

Com as crescentes indicações para cirurgia no paciente com câncer, Vizzotto e Soares avaliaram a cicatrização das anastomoses após a injeção de quimioterápicos intraperitoneais e concluíram não haver diferenças significativas entre os grupos estudados ${ }^{50,51,58,59}$. Ainda se falando de pacientes oncológicos, Ferreira, em estudo experimental concluiu que radioterapia pré-operatória e pós-operatória diminui a força necessária à ruptura total das anastomoses colônicas e a área percentual ocupada pelo colágeno tipo I (maduro) no $4^{\circ}$ e $8^{\circ}$ dia de pós-operatório ${ }^{22}$.

A desnutrição protéica pode prejudicar a cicatrização de feridas por prolongar a fase inflamatória, diminuir a síntese e a proliferação fibroblástica, angiogênese e síntese de colágeno e proteoglicanos. Pode ainda reduzir a força tênsil de feridas, limitar a capacidade fagocítica de leucócitos e aumentar a taxa de infecção de feridas ${ }^{25,37,45}$. Em 2004, Coelho-Lemos et a ${ }^{16}$ publicaram um estudo que avaliou a cicatrização de ratos neonatos de matrizes desnutridas e concluíram que a desnutrição intra-útero reduz a força na ferida abdominal desses ratos e que a deposição de colágeno é maior nestes animais, sugerindo que a ferida seja prioridade biológica ${ }^{16,17}$. Gonçalves et al. ${ }^{24}$ demonstraram, em 2005, que a desnutrição reduz a força de ruptura e a porcentagem de deposição de colágeno maduro nas anastomoses colônicas no $5^{\circ}$ dia do pós-operatório. Também que a realimentação pré-operatória por sete dias aumenta 
a força de tração e a porcentagem de colágeno maduro ${ }^{24}$. Complementando o estudo de Gonçalves, Schmitz, em 2006, avaliou o efeito da suplementação com glutamina na cicatrização de anastomoses colônicas e concluiu que a suplementação leva a melhora da cicatrização com aumento da força máxima de tração e da deposição de colágeno tipo $\mathrm{III}^{46}$.

Partindo do princípio da manipulação nutricional, diversos estudos foram realizados com fitoterápicos, mostrando seus efeitos na cicatrização. Dentre os fitoterápicos estudados, podem ser citar a Passiflora edulis (maracujá); Orbignya phalerata (babaçu) e a Jotropha gossypiifolia L.(pião roxo) e a Schimus terebinthifolius raddi (aroeira). Todos mostraram, de alguma forma, melhora na cicatrização da parede abdominal, anastomoses colônicas e gástricas, mediante avaliação histológica e ou da força de ruptura ${ }^{5,7,23,57}$.

\section{Medidas de avaliação da cicatrização}

\section{Tensiometria}

Em 1853, Paget, introduziu a medida de força tênsil de tecidos ao seccionar e reparar cirurgicamente tendões de coelhos ${ }^{6}$. Mediante tecnologia rudimentar observou que o segmento reparado ganhava força ao longo do período pós-operatório. Posteriormente, em 1929, Howes et $\mathrm{al}^{27}$ sistematizaram a técnica de medida da força tênsil através de dispositivo mecânico (tensiômetro) que apresentava resultados reprodutíveis ${ }^{27}$.

O tipo de instrumento a ser utilizado pode influenciar a medida de força de ruptura. Naresse et al..$^{38}$ desenvolveram instrumento para medida da força de ruptura, o qual foi utilizado para estudo de cicatrização de anastomoses intestinais em cães. Os autores concluíram que o aparelho apresentou precisão de medida de $98 \%$, sendo considerado instrumento adequado para estudos de cicatrização intestinal $^{38}$. Diversos estudos utilizaram instrumento universal de ensaio mecânico computadorizado com garras de pressão pneumática e sistema eletrônico de aquisição de dados, o que permitiu que tração uniforme e constante fosse aplicada ao tecido a ser estudado $4,10,16,17,18,22,24,46,47,54,56,59$.

A pressão de ruptura é utilizada principalmente para testar a cicatrização de anastomoses e é realizada através da conexão de uma de suas extremidades a bomba de insuflação e fechando-se a outra. Através desta última infunde-se líquido ou ar atmosférico até que haja ruptura da anastomose. A pressão de ruptura é registrada por manômetro ou fisiógrafo. Este método pode ser aplicado ao animal vivo e anestesiado, sem se desfazerem as aderências perianastomóticas ou em segmento ressecado; os resultados serão diferentes nos dois casos, mas ambos com validade científica ${ }^{7}$.

A força de ruptura é medida pela força de tração do segmento de tecido até que ele se rompa. Fahart et al. ${ }^{21}$ desenvolveram dispositivo mecânico para efetuar a tração de um segmento de tecido e o utilizaram para avaliação de anastomoses em processo de cicatrização ${ }^{21}$.Posteriormente houve aprimoramento do método, com o desenvolvimento de um dispositivo eletro-mecânico, descrito por Jonsson et al. ${ }^{30}$, com maior precisão para a aferição da força de ruptura. A tração pode ser exercida com aumento contínuo da força ou aumentando-se a força em intervalos ${ }^{8}$.

Mais recentemente foi desenvolvido método mecânico computadorizado de alta precisão para a determinação da força de ruptura. A correlação entre este método e o de pressão de ruptura, já consagrado na literatura, demonstrou que a força mínima de tensão seria o melhor método para avaliação biológica da cicatrização das anastomoses, enquanto a de ruptura seria mais adequada na pesquisa de integridade anastomótica em geral, não refletindo o processo cicatricial ${ }^{28}$ (Figuras 2, 3 e 4).

Considerando-se o que foi exposto, hoje, os métodos de tração são considerados padrão-ouro para a avaliação da cicatrização de feridas.

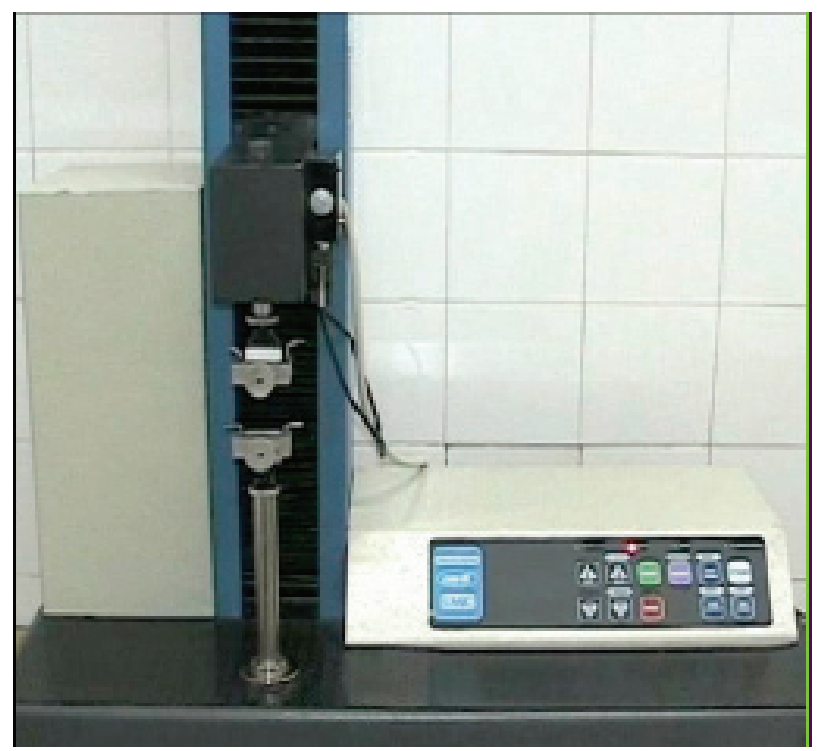

Máquina universal de ensaio mecânico computadorizada, da marca EMIC®, modelo DL-500-MF, com garras de pressão ajustáveis.

FIGURA 2 - Máquina de Tração

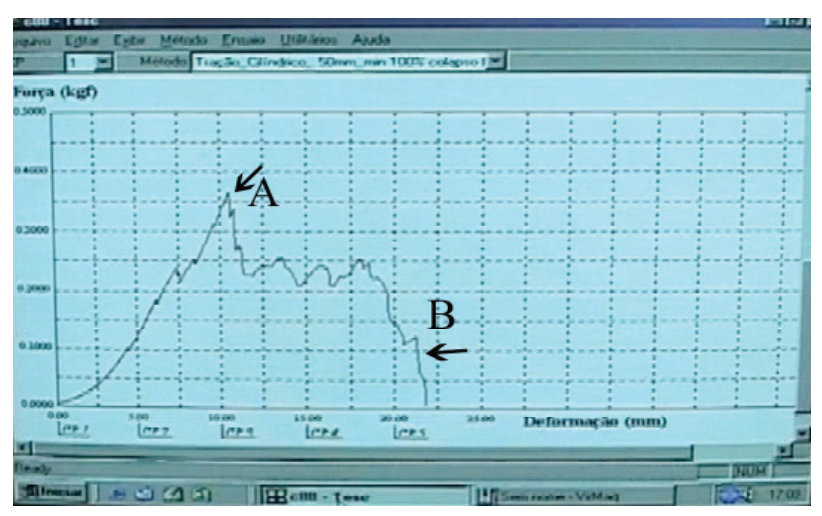

Sistema eletrônico para aquisição de dados com utilização do software TESC, versão 1.01 , onde: $\mathrm{A}=$ força máxima de tração e $\mathrm{B}=$ força de ruptura

FIGURA 3 - Gráfico de tração 


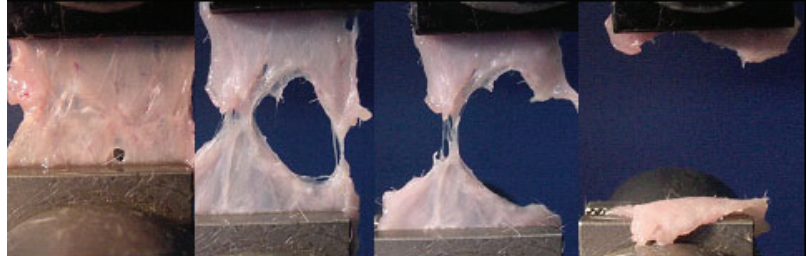

Adaptado de Coelho-Lemos, $2003 .^{17}$

FIGURA 4 - Exemplo de tração de parede abdominal de ratos lactentes

\section{Análise histológica e densitometria do colágeno}

A microscopia ótica é método de avaliação do processo de cicatrização de anastomoses intestinais muito utilizada em estudos clínicos e experimentais. Sua real importância é, de certo modo, questionada pela subjetividade na avaliação. Entretanto, quando realizada por patologistas experientes e imparciais, pode fornecer dados passíveis de quantificação que são fundamentais na análise e comparação de processos cicatriciais.

O processo de reparação pode ser avaliado mediante técnica de coloração com hematoxilina-eosina (HE), utilizando diversos indicadores tais como: exsudato neutrofílico, edema intersticial, necrose de mucosa, necrose transmural, deposição de fibrina, congestão vascular, infiltrado monomorfonuclear, atividade regenerativa da mucosa, proliferação fibroblástica, neoformação vascular, processo granulomatoso e fibrose intersticial.

Outro método histológico de grande utilidade, principalmente na avaliação e diferenciação de tipos de colágeno, é o da polarização com picrosirius. Quando o corante sirus red F3AB - corante ácido e forte -, reage com o colágeno, sua birrefringência normal é aumentada devido ao fato de suas moléculas serem acoladas às fibrilas de colágeno de maneira que elas fiquem paralelas. $\mathrm{O}$ estudo comparativo de órgãos de animais vertebrados por este método identificou estreita correlação entre a localização de diferentes cores e a intensidade de birrefringência com a distribuição bioquímica dos tipos I, II e III de colágeno, criando a possibilidade de sua diferenciação tecidual. O colágeno tipo I aparece como fibras espessas, fortemente birrefringentes, de coloração amarelo ou vermelho; já o do tipo III possui $\mathrm{o}$ aspecto de fibras finas, fracamente birrefringentes e de coloração esverdeada ${ }^{31}$. O cálculo da porcentagem das fibras, classificadas em maduras ou imaturas de acordo com a sua coloração, permite avaliação qualitativa de fibras colágenas, podendo ser utilizado em estudos de cicatrização de anastomoses intestinais ${ }^{4,10,16,17,18,22,24,46,47,48,54,56,59}$ (Figura 5).

Coelho-Lemos, em estudo para verificar a influência da desnutrição intra-uterina na cicatrização da parede abdominal em ratos lactentes, idealizou o índice de maturação do colágeno (IMaC), que foi definido como a razão entre as percentagens de colágeno tipo I e tipo $\mathrm{III}^{17}$. Este índice tem sido amplamente utilizado por ser de fácil reprodutibilidade e facilitar a interpretação dos resultados, onde valores acima de um (1) representam predominância de colágeno tipo I e os abaixo de 1 representam predomínio de colágeno tipo III ${ }^{16,17}$.

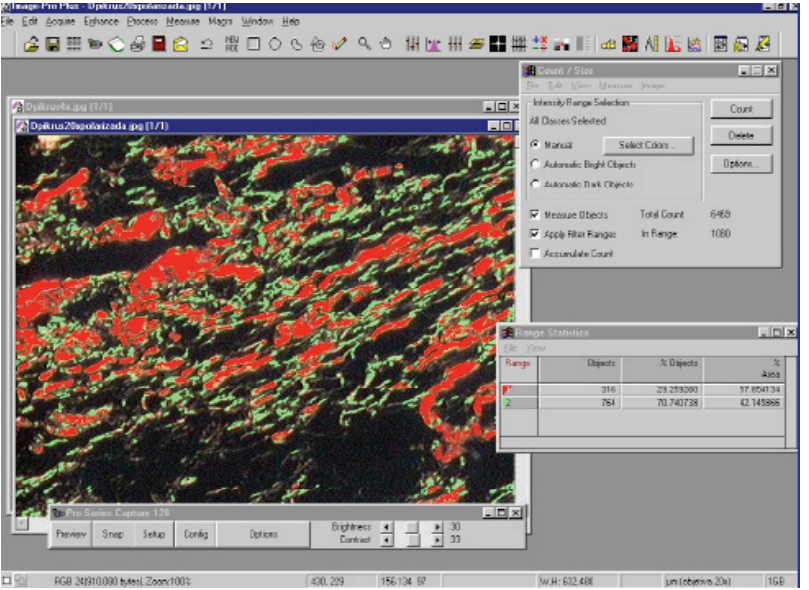

Aplicativo computadorizado utilizado para mensuração da densitometria do colágeno, onde o vermelho representa colágeno tipo I (imaturo) e o verde, as fibras de colágeno tipo III (maduro)

FIGURA 5 - Análise da densitometria do colágeno

\section{Imunoistoquímica}

A imunoistoquímica é o método diagnóstico que tem como objetivo detectar um antígeno tecidual ou celular, mediante a utilização de anticorpo específico dirigido contra este componente celular ${ }^{53}$. Consiste na utilização de anticorpos específicos conhecidos e manipulados quimicamente para que, ao se ligarem aos seus antígenos celulares ou teciduais correspondentes, devido a diversas reações e utilização de cromógenos, produzam alteração de coloração visível à microscopia óptica, a qual indica o antígeno específico e o quantifica ${ }^{60}$.

Para o estudo da cicatrização são utilizados a determinação da angiogênese e dos miofibroblastos.

A quantificação de vasos sangüíneos foi realizada por meio da técnica de imunistoquímica, utilizando-se o anticorpo antifator VIII, também conhecido como fator de von Willebrand. O fator VIII é altamente específico para marcar tecido vascular ${ }^{60}$, e foi utilizado como marcador de vascularização em vários estudos ${ }^{4,14,48}$.

A quantificação de miofibroblastos é realizada por meio de imunoistoquímica, utilizando-se o anticorpo anti$\alpha$-actina muscular lisa (actina $\alpha$-sm). Estudos comprovam a importância dos miofibroblastos no processo cicatricial e demonstraram que existe relação direta entre o nível de actina $\alpha$-sm e a capacidade contrátil dos miofibroblas$\operatorname{tos}^{26,35}$.

Estudos experimentais realizados recentemente demonstraram correlação direta entre a força máxima de tração (considerado padrão-ouro) e a quantificação de vasos e miofibroblastos na avaliação da cicatrização dos tecidos ${ }^{4,48}$.

\section{Fatores de crescimento}

A biologia molecular proporcionou a quantificação dos fatores de crescimento, através de complexas reações utilizando material genético encontrado no tecido de granulação das feridas. Em recente tese de doutorado, Pereira 
estudou os fatores de crescimento em feridas cutâneas e concluiu que sua dosagem correlaciona-se diretamente com a morfometria do colágeno ${ }^{41}$.

\section{Quelóides e cicatrizes hipertróficas}

Quelóides e cicatrizes hipertróficas são distúrbios da cicatrização, e diferenciados pelo comportamento clínico; quelóides ultrapassam os limites da incisão $0^{52}$ enquanto as cicatrizes hipertróficas permanecem confinadas aos limites da incisão ${ }^{33}$.

Ambas são decorrentes de resposta inflamatória excessiva durante a cicatrização, com perda do controle normal entre síntese e degradação ${ }^{1}$. Dentre os fatores implicados em sua patogênese incluem-se: trauma, disfunção de fibroblastos, níveis aumentados de fatores de crescimento ou outras citocinas e diminuição da apoptose. São mais comuns em negros e em orientais.

Não existem diferenças histológicas entre quelóides e cicatrizes hipertróficas, porém à microscopia eletrônica observa-se substância amorfa ao redor dos fibroblastos no quelóide ${ }^{20}$. Também há maior atividade metabólica e presença de anticorpos antinucleares nos quelóides ${ }^{55}$.

Ainda hoje o melhor tratamento para cicatrizes hipertróficas e quelóides é a prevenção. Procedimentos cirúrgicos desnecessários devem ser evitados ${ }^{49}$, e caso isso não seja possível, feridas devem ser fechadas sob mínima tensão. Também é importante salientar que cuidados locais, como hemostasia, manuseio adequado dos tecidos, uso de fios monofilamentares e desbridamento de tecidos desvitalizados, são importantes fatores preventivos.

Existem inúmeras formas de tratamento para estas condições, entre elas: ressecção cirúrgica, infiltração intralesional de corticóide, laser, uso de lâminas de silicone e betaterapia ${ }^{1,49}$. A grande quantidade de tratamentos disponíveis sinaliza a dificuldade de tratar destas condições patológicas. Freqüentemente, a ressecção cirúrgica é as- sociada a algum método adjuvante, como infiltração de corticóide e fitas de silicone.

\section{Perspectivas}

Pace determinou o efeito de substâncias antioxidantes na cicatrização de retalhos cutâneos em ratos. $\mathrm{O}$ uso de substâncias antioxidantes tem sido relacionado à menor formação de radicais livres e menor dano tecidual em situações de hipóxia. As substâncias utilizadas foram vitamina C, vitamina E e Gingko biloba. Foi comprovada maior viabilidade tecidual nos grupos que receberam as substâncias antioxidantes ${ }^{40}$. Esses resultados sugerem que outras manipulações nutricionais, metabólicas e medicamentosas deverão ser investigadas pelo seu potencial de influenciar positivamente o processo cicatricial

Recentes pesquisas têm sido direcionadas para atuar na modulação da cicatrização a nível molecular, na tentativa de reduzir cicatrizes patológicas hipertróficas ${ }^{1}$.

Alguns estudos já tem investigado a utilização de terapia gênica para o tratamento das feridas ${ }^{42,43}$, com o objetivo de estimular o processo cicatricial mais rápido, com menor cicatriz e com restauração completa de pêlos e glândulas ${ }^{36}$.

\section{CONCLUSÕES}

A cicatrização é processo complexo, que começou a ser entendido em maior amplitude nos últimos anos. Contudo, ainda há necessidade de se continuar estudando seus mecanismos, porque grande parte dele ainda é desconhecida. O futuro é promissor em medidas preventivas e curativas mais eficientes e que possam estar à disposição dos cirurgiões, diminuindo assim a possibilidade de complicações no manuseio dos doentes que necessitam agressão cirúrgica para cura dos seus males.

Campos ACL, Borges-Branco A, Groth AK. Wound healing. ABCD Arq Bras Cir Dig 2007; 20(1):51-8.

ABSTRACT - Background - The wound healing consists of a perfect and coordinated cascade of events that result in tissue reconstitution. The healing process is common to all wounds, independently of the agent that has caused it. It is divided didactically into three phases: inflammation, proliferation or granulation and remodelation or maturation. The collagen is the most abundant protein in the human body and is also the main component from the matrix wounds. The collagen is organized in a thick and dynamic net, resulting from constant collagen deposition and reabsorption. The wound scar is the result of the interaction among tissue synthesis, degradation and remodeling. There are several ways to evaluate wound healing. The methods most used are: tensiometry, collagen morphometry, immunohistochemistry and more recently, the dosage of growth factors. Methods - All thesis presented at the Graduate Program in Clinical Nutrition of the Federal University of Paraná in the Research Line of "Organs and Tissue Healing" were reviewed. Additionally a literature search on the subject was undertaking by using Pubmed (www.pubmed.com) as well as Bireme (www.bireme.com.br). Conclusions - The wound healing is a complex process which has been subjected to numerous research studies in order to better understand it. Recent studies have attempted to influence the process by using either nutrients or drugs in order to achieve faster recovery after surgical trauma.

HEADINGS - Wound healing. 


\section{REFERÊNCIAS}

1. Al-Attar A, Mess S, Thomassen JM, Kauffman CL, Davison SP. Keloid pathogenesis and treatment. Plast Reconstr Surg, 2006. 117(1): p. 286-300.

2. Allen DB, Maguire JJ, Mahdavian M, Wicke C, Marcocci L, Scheuenstuhl H, Chang M, Le AX, Hopf HW, Hunt TK. Wound hypoxia and acidosis limit neutrophil bacterial killing mechanisms. Arch Surg 1997; 132(9):991-6.

3. Allendorf JD, Bessler M, Huang J, Kayton ML, Laird D, Nowygrod R, Treat MR. Helium-neon laser irradiation at fluences of 1,2 , and $4 \mathrm{~J} / \mathrm{cm} 2$ failed to accelerate wound healing as assessed by both wound contracture rate and tensile strength. Lasers Surg Med 1997; 20(3):340-5.

4. Alves, MR. Influência da nicotina, durante a gestação e lactação, na cicatrização da parede abdominal de ratos lactentes: estudo tensiométrico, mofológico e imunoistoquímico. Curitiba, 2006. Tese (Doutorado em Clínica Cirúrgica) - Setor de Ciências da Saúde, Universidade Federal do Paraná.

5. Baldez RN, Malafaia O, Czescko NG, Martins NLP, Ferreira LM, Ribas CAPM, Salles Jr. G, Del Claro RP, Santos LOM, Graça Neto L, Araújo LRR. Análise da cicatrização do colon com uso do extrato aquoso da Orbignya phalerata (babaçu) em ratos. Acta Cir Brasil. 2006; 21 (suplem 2): 31-38.

6. Ballantyne GH. Intestinal Suturing: Review of the Experimental foundations for traditional doctrines. 1983, Dis Colon Rectum; 26:836-43.

7. Bezerra JAF; Campos ACL; Vasconcelos PRL; Nicareta JR; Ribeiro GR; Sebastião APM; Urdiales AIA; Moreira M; Borges AM. Extrato de Passiflora edulis na cicatrização de anastomoses colônicas em ratos: estudo morfológico e tensiométrico. Acta Cir Bras. 2006; 21(suplem 3): 16-25.

8. Blomquist P, Jiborn H, Zederfeldt B. The effect of relative bowel rest on collagen metabolism and suture holding capacity in the colonic wall. Res. Exp. Med. Berl. 1984; 184:221-6.

9. Bonin EA, Campos ACL, Coelho JCU, Matias JEF, Malafaia O, Jonasson TH. Effect of pantoprazole administered subcutaneously on the healing of sutured gastric incisions in rats. Eur Surg Res. 2005; 37: 250-56.

10. Bonin, EA. Supressão ácida por pantoprazol e cicatrização de sutura gástrica em ratos. Curitiba, 2003. Dissertação (Mestrado em Clínica Cirúrgica) - Setor Ciências da Saúde, Universidade Federal do Paraná.

11. Broughton G, 2nd, Janis JE, Attinger CE. The basic science of wound healing. Plast Reconstr Surg 2006; 117(7 Suppl):12S-34S.

12. Broughton G, 2nd, Janis JE, Attinger CE. Wound healing: an overview. Plast Reconstr Surg 2006; 117(7 Suppl):1e-S-32e-S.

13. Carrel A. The treatment of wounds. JAMA. 1910; 55:2148.

14. Chibata, M. Nicotina subcutânea e cicatrização da parede abdominal em ratos: estudo histológico e tensiométrico. Curitiba, 2003. Dissertação (Mestrado em Clínica Cirúrgica) - Setor de Ciências da Saúde, Universidade Federal do Paraná.

15. Clark RAF: Wound repair. In: Kumar, Robbins, Cotran: Pathologic Basis of Disease, 7th ed., Ed. Saunders, p.112, 2005.

16. Coelho-Lemos ICM, Campos ACL, Almeida M, Malafaia O, Schüler SL, Gurmini J. In-Utero Malnutrition Influences Wound Healing of Newborn Rats as Measured by Tensile Strength and Collagen Deposition. JPEN J. Parent Ent Nut 2004; 28 (4): 241-4.

17. Coelho-Lemos, ICM. Influência da desnutrição intra-uterina na cicatrização da parede abdominal de ratos lactentes avaliada mediante estudo tensiométrico e da morfometria do colágeno. Curitiba, 2003. Tese (Doutorado em Clínica Cirúrgica) - Setor de Ciências da Saúde, Universidade Federal do Paraná.

18. da Costa MR, Campos ACL, Coelho JCU, de Barros AM, Matsumoto HM Oral Glutamine and the Healing of Colonic Anastomoses in Rats. J.Parent. Enteral.Nutr. 2003; 27(3):182-6.

19. Deodhar AK, Rana RE. Surgical physiology of wound healing: a review. J Postgrad Med 1997; 43(2):52-6.

20. Ehrlich HP, Desmouliere A, Diegelmann RF, et al. Morphological and immunochemical differences between keloid and hypertrophic scar. Am J Pathol 1994; 145(1):105-13.

21. Farhat SM; Amer NS; Weeks DS; Musselman MM. Effect of mechlorethamine (nitrogen mustard) on healing of abdominal wounds. Arch Surg. 1958; 76:74953.

22. Ferreira, M. Radioterapia pré e pós-operatória na cicatrização de anastomoses colônicas em ratos avaliada mediante estudo tensiométrico, histológico e da morfometria do colágeno. Curitiba, 2004. Dissertação (Mestrado em Clínica Cirúrgica) - Setor de Ciências da Saúde, Universidade Federal do Paraná

23. Gomes CS, Campos ACL, Torres OJM, Vasconcelos PRL, Moreira ATR, Tenório SB, Tâmbara EM, Sakata K, Moraes Jr. H, Ferrer, ALS. Efeito do extrato de Passiflora Edulis na cicatrização da parede abdominal de ratos: estudo morfológico e tensiométrico. Acta Cir Brasil. 2006; 21 (suplem 2): 9-16.

24. Gonçalves CG, Campos AC, Groth AK, Ferreira M, Coelho, JCU, Meguid, MM. Influence of Preoperative Feeding on the Healing of Colonic Anastomosis in Malnourished Rats. JPEN J Parenter Enteral Nutr. 2005;29(1 Suppl): 1-48.

25. Haydock DA; Flint MH; Hyde KF; Reilly HC; Poole CA; Hill GL. The efficacy of subcutaneous gortex implants in monitoring wound healing response in experimental protein deficiency. Connect Tissue Res. 1988; 17: 159-69.

26. Hinz B; Mastrangelo D, Iselin CE, Chaponnier C, Gabbianni G. Mechanical tension controls granulation tissue contractile activity and myofibroblast differentiation. Am J Pathol. 2001; 159 (3): 1009-20.
27. Howes EL, Sooy JW, Harvey SC. The healing of wounds as determined by their tensile strength. JAMA. 1929; 92(1):42-45

28. Ikeuchi D, Onodera H, Aung T, Kan S, Kawamoto K, Iamamura M, Maetani S. Correlation of tensile strength with bursting pressure in the evaluation of intestinal anastomosis. Dig. Surg. 1999; 16:478-85.

29. Jibon $\mathrm{H}$; Ahonen J; Zederfeldt B. Healing of experimental colonic anastomoses IV. Effect of suture technique on collagen metabolism in the colonic wall. Am J Surg, 1980; 139: 406-13.

30. Jonsson K, Jiborn H, Zederfeldt B. Breaking strength of small intestinal anastomoses. Am. J. Surg. 1983; 145:800-3.

31. Junqueira LCU, Montes GS, Sanches EM. The influence of tissue section thickness on the study of collagen by the Picrosirius-Polarization Method. Histochemistry, 1982; 74:153-6.

32. Lawrence WT, Diegelmann RF. Growth factors in wound healing. Clin Dermatol 1994; 12(1):157-69.

33. Li W, Dasgeb B, Phillips T, Li Y, Chen M, Garner W, Woodley DT. Woundhealing perspectives. Dermatol Clin 2005; 23(2):181-92.

34. Longaker MT, Peled ZM, Chang J, Krummel TM. Fetal wound healing: progress report and future directions. Surgery 2001; 130(5):785-7.

35. Lorena D, Uchio K, Costa AM, Desmoliere A. Normal scarring: importance of myofibroblasts. Wound Repair Regen. 2002; 10 (2): 86-92.

36. Martin P. Wound healing - aiming for perfect skin regeneration. Science 1997; 276(5309):75-81.

37. Mayes T; Gotshemitch MM. Burns and wound healing. In: Matarase LE; Gottschilich MM. Contemporary Nutrition Support Practice - Clinical Guide. 2ed. New York: Sauders. 1998; 595-615.

38. Naresse LE, Mendes EF, Curi PR, Lucchiari PH, Kobayasi S. Aparelho para medida da força de ruptura das anastomoses intestinais. Rev. Hosp. Clin. Fac. Med. S. Paulo. 1987; 42(5): 204-8.

39. Orgill D, Demling RH. Current concepts and approaches to wound healing. Crit. Care Med. 1988:16(9): 899-908.

40. Pace DT. Efeito de substâncias antioxidantes (Vitamina C, Vitamina E e Gingko biloba) na viabilidade de retalho cutâneo dorsal em ratos. Curitiba, 2003. Dissertação (Mestrado em Clínica Cirúrgica) - Setor de Ciências da Saúde, Universidade Federal do Paraná.

41. Pereira, CCA. Influência da dieta enteral suplementada com arginina e antioxidantes sobre a cicatrização cutânea experimental. São Paulo, 2006. Tese (Doutorado em Ciências) - Universidade de São Paulo.

42. Petrie NC, Vranckx JJ, Hoeller D, et al. Gene delivery of PDGF for wound healing therapy. J Tissue Viability 2005; 15(4):16-21.

43. Petrie NC, Yao F, Eriksson E. Gene therapy in wound healing. Surg Clin North Am 2003; 83(3):597-616, vii.

44. Robson MC; Steed DL; Franz MG. Wound healing: biologic features and approaches to maximize healing trajectories. In: Robson MC; Steed DL; Franz MG. Wound healing. Curr Probl Surg. Chicago, 2001; 38(2): 73-94.

45. Ruberg RL. Role of nutrition in wound healing. Symposium on Wound Management. Surg Clin North Am. 1984; 64: 705-14.

46. Schmitz LD. Nutrição perioperatória suplementada com glutamina em ratos desnutridos submetidos à anastomose colônica: avaliação morfológica e tensiométrica. Curitiba, 2006. Dissertação (Mestrado em Clínica Cirúrgica) - Setor de Ciências da Saúde, Universidade Federal do Paraná.

47. Skinovsky JA. A influência da nicotina na cicatrização de anastomoses do intestino delgado em ratos. Curitiba, 2000. Dissertação (Mestrado em Clínica Cirúrgica) - Setor de Ciências da Saúde, Universidade Federal do Paraná.

48. Skinovsky, JA. A influência da nicotina na cicatrização de anastomoses do intestino delgado em ratos: angiogênese e miofibroblastos. Curitiba, 2005. Tese (Doutorado em Clínica Cirúrgica) - Setor de Ciências da Saúde, Universidade Federal do Paraná.

49. Slemp AE \& Kirschner RE. Keloids and scars: a review of keloids and scars, their pathogenesis, risk factors, and management. Curr Opin Pediatr, 2006. 18(4): p. 396-402.

50. Soares EWS, Campos ACL, Matias JEF, Coelho JCU, Malafaia O. Influência da quimioterapia pós-operatória com 5 -fluorouracil na cicatrização de anastomoses colônicas em ratos. ABCD - Arquivos Brasileiros de Cirurgia Digestiva. 2002; 15(1): 31-35.

51. Soares EWS. Efeito do uso pós-operatório de 5-fluorouracil na cicatrização de anastomoses colônicas em ratos. Curitiba, 2001. Dissertação (Mestrado em Clínica Cirúrgica) - Setor de Ciências da Saúde, Universidade Federal do Paraná.

52. Steinbrech DS, Longaker MT, Mehrara BJ, et al. Fibroblast response to hypoxia: the relationship between angiogenesis and matrix regulation. J Surg Res 1999; 84(2):127-33

53. Torres, LFB, Sluminsky, BG, Tirono, FA, Noronha L, Giron RL, Telles, JEQ. Contribuição da imunoistoquímica em patologia cirúrgica: experiência de 10 anos. Rev Med Paraná. 1998; 56 (2): 31-38.

54. Trubian, PS. Octreotide subcutâneo e cicatrização de sutura gástrica em ratos: estudo tensiométrico e da morfometria do colágeno. Curitiba, 2004. Dissertação (Mestrado em Clínica Cirúrgica) - Setor de Ciências da Saúde, Universidade Federal do Paraná. 
55. Ueda K, Furuya E, Yasuda Y, et al. Keloids have continuous high metabolic activity. Plast Reconstr Surg 1999; 104(3):694-8.

56. Urdiales, AIA. Octreotide na cicatrização de anastomoses colônicas de ratos. Curitiba, 2006. Dissertação (Mestrado em Clínica Cirúrgica) - Setor de Ciências da Saúde, Universidade Federal do Paraná.

57. Vale JR, Czeczko, NC, Aquino JU, Ribas-Filho JM, Bettega L, Vasconcelos, PRL, Correa Neto, MA, Nassif PAN, Mazza M, Henriques GS. Estudo comparativo da cicatrização de gastrorrafias com e sem o uso do extrato de Jatropha gossypiifolia L. (pião roxo) em ratos. Acta Cir Brasil. 2006; 21 (suplem 3): 40-48.

58. Vizzotto Jr, AO, Noronha L, Scheffel DLH, Campos ACL. Influência da cisplatina administrada no pré e no pós-operatório sobre a cicatrização de anastomoses colônicas em ratos. J Bras Patol Med Lab. 2003; 39(2):143-9.
59. Vizzotto Jr, AO. Influência da cisplatina administrada no pré e pós-operatório sobre a cicatrização de anastomoses colônicas em ratos testada pela força de resistência à tração. Curitiba, 2001. Dissertação (Mestrado em Clínica Cirúrgica) - Setor de Ciências da Saúde, Universidade Federal do Paraná.

60. Werner, B. Indicações e contribuição diagnóstica da imunoistoquímica em patologia cirúrgica. Curitiba, 2002. Dissertação (Mestrado em Clínica Cirúrgica) - Setor de Ciências da Saúde, Universidade Federal do Paraná.

61. Witte MB \& Barbul A. General principles of wound healing.Surg Clin North Am. 1997; 77(3):509-28.

Conflito de interesse: não há Fonte financiadora: não há Recebido para publicação em: 08/08/2006 Aceito para publicação em: 21/11/2006 